\title{
Le mythe du consensus
}

Le rôle des enseignants dans les réformes scolaires dans le contexte suisse

The end of consensus-based management. Consultation of teachers in education reform in Switzerland

El mito consensual. El papel de los docentes en las reformas en Suiza

Danièle Périsset-Bagnoud

\section{OpenEdition}

1 Journals

Édition électronique

URL : https://journals.openedition.org/ries/1899

DOI : 10.4000/ries. 1899

ISSN : 2261-4265

Éditeur

France Education international

Édition imprimée

Date de publication : 1 septembre 2002

Pagination : 59-68

ISBN : 978-285420-554-1

ISSN : 1254-4590

Référence électronique

Danièle Périsset-Bagnoud, « Le mythe du consensus », Revue internationale d'éducation de Sèvres [En ligne], 30 | septembre 2002, mis en ligne le 24 novembre 2011, consulté le 08 juillet 2021. URL : http:// journals.openedition.org/ries/1899; DOI : https://doi.org/10.4000/ries.1899

(c) Tous droits réservés 


\title{
Le mythe du consensus
}

\section{Le rôle des enseignants dans les réformes scolaires dans le contexte suisse}

\author{
Danièle Périsset-Bagnoud
}

\section{LE SYSTÈME FÉdÉRAL SUISSE}

Écrire un bref article à propos du rôle des enseignants suisses dans le contexte actuel des rénovations et de l'évolution de la profession dans ce petit pays relève de la gageure. En effet, cette entité politique, au territoire peu étendu, relève de la fédération historique de nombreuses régions. Historiquement, sous la pression des diverses guerres européennes, ces dernières s'assemblent entre 1291 et 1815 et forment la Confédération helvétique tout en conservant leur souveraineté politique.

Or donc, à ce jour, la Suisse compte 20 cantons plus 6 demi-cantons, soit, au total, 26 systèmes scolaires différents. Quelques tentatives de centralisation ont eu lieu, aux XVIII et XIX ${ }^{\mathrm{e}}$ siècles : l'enquête Stapfer menée en 1798 (voir Boucard, 1938; Rohr, 1998), pour des raisons politiques mais aussi à cause de la forte résistance déployée par les cantons s'estimant peu concernés par la question scolaire, n'a pu aboutir; un siècle plus tard, une nouvelle tentative de centralisation scolaire (instauration d'un secrétariat contrôlant une législation fédérale scolaire) est fortement combattue dans les cantons conservateurs, officiellement à propos de la question religieuse (voir notamment Bioley, 1882). Pourtant, des rencontres entre chefs des départements de l'Instruction publique sont mises sur pied dès 1874, malheureusement sous une forme dont peu de traces nous sont parvenues (Forster, 1999). Il faut attendre encore un siècle pour voir aboutir une réelle coordination scolaire. En 1970, un concordat est signé par les cantons et approuvé par le Conseil fédéral. Une institution intercantonale de droit publique (la Conférence des directeurs de l'instruction publique, $\operatorname{CDIP}^{1}$ ) est ainsi créée "aux fins de développer l'école et d'harmoniser leurs législations cantonales respectives » (art. 1, but du concordat). Les législations scolaires des cantons signataires sont en effet coordonnées à propos de l'âge d'entrée et de la durée de la scolarité obligatoire, des dates auxquelles les rentrées scolaires ont annuellement lieu. Pour les autres objets relatifs à la politique éducative dont chacun conserve la libre gestion, la CDIP élabore des recommandations, par exemple à propos des plans d'études, du matériel

1. Pour toute information relative aux législations et recommandations émanant de cet organe, consulter son site http://www.cdip.ch/f 
d'enseignement, du libre passage entre écoles équivalentes ou encore de la reconnaissance mutuelle des diplômes de fin d'études.

Enfin, et afin de faciliter la coordination entre cantons, ceux-ci ont la possibilité de se regrouper en quatre Conférences régionales. Libres de leur choix, les cantons décident eux-mêmes de leur adhésion aux dites conférences, selon leurs affinités géographiques et culturelles (Suisse romande et Tessin, Suisse du nord-ouest, Suisse centrale, Suisse orientale).

C'est dire la pointilleuse autonomie que conserve chaque canton au niveau de l'organisation de sa structure scolaire, de sa législation, des réformes engagées, à l'écart de celles conduites par son voisin.

\section{UNE (R)ÉVOLUTION DES SYSTĖMES CANTONAUX DE FORMATION}

Si les Écoles normales devaient leur relative connivence idéologique aux conditions politiques entourant leur émergence au XIX ${ }^{\mathrm{e}}$ siècle (voir notamment Périsset Bagnoud, 2000 ; Criblez, Hofstetter \& Périsset Bagnoud, 2000), leur évolution, notamment dans la seconde moitié du $\mathrm{XX}^{\mathrm{e}}$ siècle, les voit poursuivre chacune sa propre mission, améliorer indépendamment leur curriculum tout en conduisant les négociations bilatérales utiles. Enfin, en 1993, les thèses que publie la CDIP concernant la promotion des Hautes écoles pédagogiques (HEP, dossier 24) donnent le coup d'envoi d'une certaine coordination en matière de formation des enseignants. Jusqu'à présent, la reconnaissance des diplômes et brevets d'enseignement tenait davantage de la bonne volonté des autorités et du marché de l'emploi. La publication du dossier 24 encourage la mise sur pied concomitante des HEP ; la reconnaissance mutuelle des diplômes d'enseignement soutient leur assujettissement aux règles intercantonales. Ainsi, les cantons sont poussés, bon gré mal gré, selon la force de l'héritage institutionnel et des représentations liées à l'enseignement, à obéir à certaines règles harmonisant les structures de formation ${ }^{2}$. Les conditions d'admission, la durée de la formation, les contenus du plan d'étude, l'articulation entre formation pratique et formation théorique, la durée des stages, la place de la recherche, les qualifications requises pour le personnel enseignant dans les HEP imposent des innovations structurelles majeures, notamment pour les établissements ayant conservé une formation intégrant formation générale et professionnelle en École normale au degré secondaire (niveau baccalauréat) ou encore pour les instituts formant les maîtresses des classes du degré préscolaire, jusque-là généralement discriminées puisque recrutées et formées à des conditions moins exigeantes que pour le degré primaire (voir à ce propos Gnaeggi, 1993).

2. Voir les Instructions pour l'élaboration d'une demande de reconnaissance des diplômes des hautes écoles pour les enseignantes et enseignants des degrés primaire et préscolaire, juillet 2000 : http://www.cdip.ch/ PDF_Downloads/Diplomanerkennung/DAnleitung_f.pdf 


\section{L’accès au métier}

Avec la publication du règlement concernant la demande de reconnaissance des diplômes HEP sur le plan national, l'accès au métier d'enseignant en Suisse, malgré les différences cantonales subsistantes, peut être profilé. Sont admis sans autre procédure dans les Hautes Écoles et pour les voies de formation conduisant aux diplômes d'enseignement pour les degrés préscolaire et primaire, les titulaires d'une "maturité gymnasiale ${ }^{3}$ ou porteurs de titres équivalents permettant l'admission dans les universités. Sont admis à la formation conduisant à l'enseignement dans le degré préscolaire uniquement les titulaires de diplômes d'écoles du degré secondaire II, écoles dites "de degré diplôme " (EDD) en trois ans. Cette dernière possibilité n'est pas utilisée en Suisse romande, puisque toutes les HEP et l'Université de Genève délivrent des titres couvrant au moins quatre années d'enseignement (le plus souvent de $1^{\text {re }}$ enfantine à $2^{\mathrm{e}}$ primaire, puis de $3^{\mathrm{e}}$ à $6^{\mathrm{e}}$ primaire; à Genève de $1^{\mathrm{E}}$ à $6^{\mathrm{P}}$ ).

Peuvent en outre être admis à la formation pour les degrés préscolaire et primaire, et sous réserve de compléments en matière de culture générale, les titulaires de maturités professionnelles ${ }^{4}$ ou de diplômes EDD. Pour cette catégorie de candidats, les HEP romandes (soit Fribourg, Valais, Vaud, Berne francophone, Jura, Neuchâtel) et quelques cantons alémaniques (Bâle, Grisons, Rorchach-St Gall) ont organisé des cours préparatoires et conditionné l'entrée desdits candidat-es à la réussite de ces cours spécifiques ou d'un examen d'entrée. Cependant, les autres HEP de Suisse alémanique (Aarau, Berne, Soleure, Thurgovie, Suisse centrale, Zürich) acceptent sans autre formalité, sinon la procédure usuelle d'admission, les candidats titulaires de diplômes qui ne permettent pas l'entrée à l'université (situation publiée par la CDIP sur son site $^{5}$ au printemps 2002).

Dans cette question de l'admission à la formation, le consensus nécessaire à l'adoption d'un règlement reconnu par tous les cantons a donc laissé une grande liberté de manœuvre à chacun. Les régions les plus attachées aux anciennes formations en École normale, dont les établissements privés religieux alémaniques encore très actifs, peuvent ajuster leurs exigences aux présupposés qui prévalent en matière de recrutement des candidats, notamment dans le déni de la pertinence d'une formation générale et professionnelle poussée pour les enseignants des premiers degrés de la scolarité. Ces cantons y trouveront vraisemblablement un bénéfice à plusieurs niveaux. D’abord, dans le profil des candidates recrutées, qui enseigneront peu, juste le temps de fonder leur propre

3. Maturité gymnasiale : diplôme de fin d'études secondaires pour les élèves issus du « gymnasium » (équivalent du lycée français).

4. Ce cursus de formation est consécutif à une formation complémentaire à un apprentissage en entreprise. Les étudiants titulaires d'une maturité professionnelle peuvent entrer dans les Hautes écoles spécialisées de niveau universitaire.

5. http://www.edk.ch/f/CDIP/Geschaefte/mainAktivit_f.html 
famille, justifiant politiquement et économiquement un cursus d'études moins long. Puis, sur le plan syndical, ce dispositif moins exigeant de recrutement favorise aussi certainement le maintien d'une différence salariale notable entre enseignants du degré préscolaire et enseignants des degrés subséquents.

Les HEP suisses étant soit très jeunes, soit encore à l'état de projet, il est encore difficile de dire ce que seront, dans les faits, les autres différences régionales entre formation théorique et formation pratique, entre procédures évaluatives et certificatives : relevons simplement que le règlement de reconnaissance précédemment cité impose aux institutions de fournir entre 20 et $30 \%$ de stage à leurs étudiants, et que les enseignants des Hautes écoles devront être munis d'un titre d'une Haute École, du moins en ce qui concerne les enseignements issus des sciences de l'éducation. Les cantons conservent encore plus de latitude pour ce qui concerne les enseignements didactiques: ici encore, les différences culturelles entre romands et alémaniques sont fortes. Pour les premiers, les didactiques relèvent du domaine de la recherche et sont bien implantées dans les universités alors que, pour les seconds, les didactiques relèvent encore souvent des "méthodologies » en cours dans les Écoles normales et sont confiées à des praticiens méritants attestant d'une formation continue et non d'un diplôme universitaire comme leurs collègues des sciences de l'éducation.

\section{L'ÉVOLUTION DES PRATIQUES ET DES CONTENUS}

Perrenoud (1998) le démontre: la synchronisation entre réformes scolaires et rénovations de la formation des enseignants, malgré l'apparentement intellectuel des deux propositions, reste difficile, introuvable, dit-il, "d'ordre mythique ». L'évolution des pratiques renvoie à l'acte quotidien de l'enseignement et appartient au curriculum dit réel ${ }^{6}$; celle des contenus, quant à elle, chevauche l'injonction institutionnelle (définition des plans d'étude et programmes - curriculum formel) et les choix professionnels (curriculum réel).

\section{Comment faire évoluer les pratiques des enseignants?}

L'impulsion première, celle qui fait évoluer les pratiques, reste difficile à cerner : est-elle issue de la passion pour l'enseignement qu'affichent nombre d'enseignants? de recherches particulièrement pertinentes ? de structures de formation efficaces? d'injonctions institutionnelles ? En Suisse, comme ailleurs, les raisons de l'évolution des pratiques sont au croisement de tensions individuelles et collectives, à la rencontre de jeux de pouvoir, d'enjeux politiques, sociaux, syndicaux, professionnels, individuels. Si les enseignants ont plus ou

6. Selon les distinctions introduites par Perrenoud, 1993. 
moins acquis le libre choix des moyens pédagogiques utilisés en classe, leur formation, les facilités d'accès à certains manuels et les conseils de l'autorité scolaire conditionnent ce choix dans une mesure certaine. D'autres facteurs, structurels, entrent encore en jeu.

En effet, hors des projets de réformes des institutions de formation des enseignants, plusieurs cantons ont tenté d'orienter les pratiques enseignantes en réformant les bases légales qui organisent l'école publique. Nous citerons, pour la Romandie, la loi dite EVM (école vaudoise en mutation) dans le Canton de Vaud (adoptée en 1996), celle dite Éducation 2000 (refusée en votation populaire en 1998) en Valais, enfin, à Genève, la réforme de l'école enfantine et primaire, initiée en 1994 et dont la généralisation imposée à toutes les classes du canton est décidée pour 2000 (voir GPR, 1999).

Lorsqu'elles n'ont pas abouti, comme en Valais, ces propositions légales ont, au moins, eu le mérite d'attirer l'attention des enseignants sur la nécessaire évolution du métier. Il est certain que la perspective de l'obligation légale de certains dispositifs, tels les cycles d'apprentissage, a soutenu les plus novateurs dans leurs initiatives et déstabilisé les plus timorés, les incitant à considérer autrement les concepts innovants organisant les apprentissages. Cependant, l'échec de la votation, dans un mouvement de balancier, a ensuite conforté les sceptiques dans leurs routines. Les audacieux ont vu leur enthousiasme étouffé ; discrètement, ils ont retrouvé l'espace de leur classe et leur relative solitude pour la mise en œuvre des dispositifs dont ils avaient cru, l'espace de quelques semaines, qu'ils allaient être imposés par l'autorité, reconnaissant ainsi publiquement leurs efforts pédagogiques.

C'est que les pratiques novatrices et les attitudes les plus ouvertes ne découlent ni de lois sophistiquées, ni d'un engagement militant, aussi enthousiaste soit-il. L'émergence remarquée, à Genève, d'un groupe de professionnels ${ }^{7}$ ouvertement opposés à l'extension de la réforme montre bien à quel point l'innovation ne peut être imposée par la seule autorité politique sans autre forme d'appropriation par les gens du terrain. L'innovation pédagogique ne peut se décréter ni se transmettre en formation initiale sans risque de déconstruction ultérieure en raison, selon l'expression de Gather Thurler (2000), de « la contre-socialisation subie au contact des collègues déjà en place ». Pourtant, le soutien de l'autorité est absolument nécessaire afin de légitimer l'innovation et de permettre son développement lorsqu'elle est décidée dans le cadre des politiques scolaires. L'innovation à grande échelle résulte d'une négociation entre le terrain de l'enseignement, le champ de la recherche pédagogique et celui des politiques éducatives, dans une alchimie si complexe (voir Gather Thurler, 1998, 2002) qu'il est finalement difficile d'en identifier les ingrédients précis, ni leur dosage, sauf à faire preuve de naïveté.

7. ARLE (Association refaire l'école), hors Société pédagogique genevoise (SPG). 


\section{L'innovation institutionnelle : un défi impossible?}

Si les méthodes d'enseignement utilisées en classe restent, en général, du ressort de l'enseignant, la question des contenus à enseigner appartient au curriculum formel et, en tant que tel, dépend de l'autorité politique responsable. Le système fédéral suisse impose donc autant de cadres législatifs et plans d'étude que de cantons. Les efforts romands de coordination ont cependant produit, en mars 1989, un plan général d'études (GRAP, groupe romand d'accompagnement des programmes). Dix ans plus tard, une nouvelle conception d'ensemble est proposée. Un travail de définition, d'élaboration et de rédaction d'un plan d'études cadre romand (PECARO) pour toute la scolarité obligatoire, y compris le niveau préscolaire, débute. Une organisation sophistiquée est mise en place. Elle tente de tenir compte à la fois des impératifs politiques - la responsabilité et l'autonomie cantonales, de la définition spécifique et de l'articulation des contenus comme de l'organisation scolaire, des enjeux théoriques contemporains et de la réalité du terrain $^{8}$.

Deux cent cinquante enseignants sont donc engagés dans ce travail formellement initié en 2000 et qui devrait s'achever en 2003 pour être soumis à la décision politique des Chefs de département en 2004. Ces professionnels, issus des Départements, d'institutions de formation ou encore directement du terrain, se répartissent en quatre groupes aux mandats bien définis ${ }^{9}$. La commission pédagogique, permanente, réunit vingt représentants des Départements de l'instruction publique du Suisse romande et $\mathrm{du}$ Tessin. Elle est appelée à se prononcer sur les propositions émises. Le comité de rédaction est composé, lui, de sept membres qui sont directement engagés par la CIIP de Suisse romande et du Tessin. Ce comité de rédaction est soutenu par un groupe noyau. Ce groupe dispose d'une large compétence collective puisque formé d'une cinquantaine de praticiens, de didacticiens et de formateurs appelés par leurs services cantonaux de l'enseignement. Le travail de ce groupe est important puisqu'il lui revient de formuler les objectifs d'enseignement et d'apprentissage devant figurer dans le plan d'études cadre. Plus largement, un groupe forum, composé de cent cinquante personnes officiellement déléguées par les Départements et les associations faîtières d'enseignants et de parents, entre en fonction dans un second temps. Censés représenter les attentes des futurs utilisateurs, leur diversité et leurs intérêts hétérogènes, les membres du groupe forum agis-

8. Comité de rédaction du «PECARO », État des travaux en février 2001, Les contours du plan d'études cadre romand, p. 2.

9. Voir le document diffusé par le Comité de rédaction du « PECARO », État des travaux en octobre 2001, Le mode d'élaboration du plan d'études cadre romand. 
sent selon un processus de consultation interne de mise en débat des options choisies. Enfin, un groupe d'experts, constitué de spécialistes de l'ensemble des disciplines et domaines d'apprentissage, est sollicité en tant que référence extérieure afin d'alimenter la réflexion et les choix du groupe noyau.

Apparemment, toutes les garanties sont prises afin d'obtenir le plus d'avis possibles, de ne négliger ni institution, ni région, ni association professionnelle. Cependant, alors que l'objectif avoué de l'opération est de "servir avant tout d'instrument de référence ${ }^{10}{ }^{0}$, de multiples questions se posent. Ainsi, deux « grands » cantons, Vaud et Genève viennent de publier leur propre programme régional. Comment les insérer dans PECARO, ou, autrement dit, comment s'assurer que PECARO sera suffisamment large pour contenir leurs spécificités, sans desservir cependant l'esprit de l'entreprise romande ni empiéter sur la souveraineté des autres cantons, ni refaire un lourd travail qui a déjà été accompli ? Chercher un consensus aussi large, n'est-ce pas affaiblir d'entrée le projet? Autre question sensible : lorsque le plan cadre sera adopté par les instances politiques cantonales, les praticiens se souviendront-ils que ce sont leurs pairs qui en ont élaboré les propositions? Lorsque l'on aborde une question aussi délicate que celle de l'orientation et de l'injonction officielles sur les questions de l'organisation des pratiques scolaires, peut-on se passer d'une formation et d'un accompagnement de ceux qui sont dans les classes (aucun canton ne semble, pour l'instant, y avoir songé), sinon lorsque les enjeux n'ont pas de répercussion concrète - ce qui n'est assurément pas le cas d'un plan d'études cadre?

Les réponses qui seront apportées à ces questions, et ce qu'en feront les enseignants, débordent le cadre régional pour rejoindre la problématique de l'évolution des pratiques scolaires. Les innovations proposées favoriseront leur développement ou, au contraire, augmenteront la résistance au changement. Car, ainsi que le rappelle Gather Thurler (2000, p. 4) citant Strittmatter, "il est impossible de mettre en œuvre des innovations contre la volonté des enseignants - forts d'une expérience longue de cent cinquante ans ceux-ci ont développé une habileté tout à fait étonnante leur permettant - au vu et su de leur entourage - d'absorber et de rendre inoffensives les nouveautés théoriques et pratiques, indépendammnent de leurs concepteurs et/ou promoteurs». Pourtant, malgré la lucidité de ces propos, nul ne peut prouver non plus que les stratégies immédiates de neutralisation mises en œuvre, individuellement ou à l'échelle d'établissements, ne participeront pas à l'assimilation, à plus ou moins long terme, des principes fondant les innovations pédagogiques.

10. Comité de rédaction du «PECARO ", état des travaux en février 2001, Les contours du plan d'études cadre romand, p. 6. 


\section{LA DYNAMIQUE DE L'ACTION POLITIQUE}

Deux points de vue concurrents, et pourtant irrémédiablement complémentaires, s'affrontent :

- le regard et la légitimité politiques de l'autorité scolaire et administrative tendront à favoriser l'injonction (même paradoxale), le discours d'intention, les preuves à apporter à l'efficacité du dispositif scolaire, la gestion des deniers publics, la responsabilité stratégique - ce regard porte aussi en lui l'essentielle garantie d'une école publique unique ;

- le regard et la légitimité des professionnels se teindront, eux, de scepticisme ou d'enthousiasme, de réaction plus ou moins naïve, épidermique ou rationnelle, de soumission à l'autorité, de demandes en formation et en soutien institutionnel, de besoin d'une reconnaissance symbolique pour l'effort accompli, pour le travail considérable que toute innovation exige, pour le travail sur l'humain qui est effectué, difficile contact quotidien avec les citoyens en devenir que sont les élèves de toutes cultures qui fréquentent l'école obligatoire.

Tout cela fait des logiques d'acteurs qui se croisent, qui s'épient, se méfient, se heurtent et heurtent la position de l'autre. Il faut les forces contraires et complémentaires de l'autorité et celles des individus pour que, finalement, le mouvement s'imprime. Prost (1992, p. 209) le rappelle: «La volonté politique doit s'appuyer sur la dynamique sociale ». Les travaux en histoire de l'éducation montrent que, jusqu'à ce jour, nulle innovation n'est advenue sans une intense et longue préparation au niveau social. Tout événement n'est que le «croisement d'itinéraires possibles» (Veyne, 1971, p. 51) et l'évolution des idées précède celle des réalisations. Ce que l'école a expérimenté hier et intégré sont nos évidences actuelles, notre tradition. Les idées soustendant les principales innovations ont, au moins, un siècle ${ }^{11}$. Le temps des élites auxquelles appartiennent ceux qui promeuvent l'innovation est rapide, celui des mentalités, du public scolaire, est lent. Les réformes et innovations qui ont quelque chance d'aboutir à terme résultent de la rencontre de ces deux temps aux rythmes disjoints. Les réformes en matière de politique de l'éducation et d'instruction publique obéissent à ce principe, tant au niveau de la scolarité obligatoire qu'à celui des réformes des formations d'enseignants.

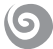

Dans ce contexte, le rôle des enseignants reste essentiel, même s'il est méconnu tant du grand public que des autorités, parce que joué par des acteurs dont la vocation n'est pas la publicité et dont le travail discret et intense au

11. (Cf. le dossier que consacre Le Monde de l'éducation aux innovateurs en novembre 2001 ; lire aussi le billet de Maulini, 2002). 
quotidien ne se clame pas aux quatre vents. Pourtant, sans le savoir ni le vouloir, les praticiens préparent le terrain des directives officielles de demain, dans un climat de négociations permanentes. Car les professionnels situent nécessairement leur action, certes à des degrés d'acceptation très variables, par rapport à l'injonction administrative et politique. En l'interprétant et en l'appliquant, les acteurs modifient ces données pensées par d'autres, leur développement et, à terme, leur intention, donnant une nouvelle orientation à l'injonction institutionnelle ultérieure, dans une interaction subtile, implicite, souvent rugueuse. La profession enseignante au quotidien des classes s'exerce, qu'on le veuille ou non, dans un contexte social, politique et culturel qui permet et dirige l'action. Ni l'une ni l'autre des parties ne peut agir seule. Toute dynamique innovante, dans le domaine des pratiques comme dans celui des curriculums, est à inscrire dans cette relation énactée, vivante, ainsi donc paradoxale, non dénuée d'ambiguïtés. Cette irrémédiable dynamique fait que, au jour le jour, l'école évolue assurément, à pas plus ou moins comptés, plus ou moins assurés, sur un chemin que seule la distance temporelle permet de mesurer.

\section{BIBLIOGRAPHIE}

BIOLEY H. (1882): Les écoles du Valais et la centralisation scolaire.

BOUCARD L. (1938): L'école primaire valaisanne à la fin du XVIII et son histoire de 1798 à 1830. Thèse présentée à la Faculté des Lettres de l'Université de Fribourg. St-Maurice. Imprimerie de l'Euvre de St-Augustin.

CRIBLEZ L., HOFSTETTER R., \& PÉRISSET BAGNOUD D. (Eds.) (2000) : La formation des enseignant(e)s primaires, Histoire et réformes actuelles. Berne : Peter Lang.

FORSTER S. (1999): Genèse de la conférence. In Politiques de l'éducation et chemins de traverse, 125 ans de collaboration intercantonale en Suisse romande. Bulletin de la CIIP, 5, 4-7. Neuchâtel : CIIP.

GATHER THURLER M. (1998): Rénovation de l'enseignement primaire à Genève: vers un autre modèle de changement. Premières expériences et perspectives, in F. CROS (dir.), Dynamiques du changement en éducation et en formation. Considérations plurielles sur l'innovation, Paris : INRP/IUFM, collection : Horizons pour la formation, pp. 229-258.

GATHER THURLER M. (2000): L'innovation négociée : une porte étroite, Revue française de pédagogie, janvier-mars 2000, n 130, pp. 29-42.

GATHER THURLER M. (2002) : Accompagner l'innovation de l'intérieur : paradoxes du développement de l'organisation scolaire, in G. PELLETIER (dir.), Le rôle de la recherche dans le changement scolaire (titre provisoire), à paraître. 
GNAEGI E. (1993): La formation des enseignants en Suisse romande et au Tessin. Conditions d'admission, durées de formation, législations et certifications. École enfantines, primaires et secondaires. Regards 93.303. Neuchâtel : IRDP.

G.P.R. (Groupe de pilotage de la rénovation) (1999): Vers une réforme de l'enseignement primaire genevois. Propositions pour la phase d'extension de la rénovation entreprise en 1994. Genève: Département de l'instruction publique, enseignement primaire.

MAULINI O. (2002): Rubrique Sacré Charlemagne, l'école, idée folle? Réformythe. L'éducateur 2.02, 22. Martigny (Suisse) : Éducateur-SER.

PÉRISSET BAGNOUD D. (2000): Vocation: régent, institutrice. Jeux et enjeux autour des Écoles normales du Valais romand de 1846 à 1994. Thèse de doctorat. Genève: Université, Faculté de psychologie et des sciences de l'éducation. [Disponible sous http://www.unige.ch/cyberdocuments/ theses2000/PerissetBagnoudD/meta.html

PERRENOUD Ph. (1998): Réformes scolaires et rénovations de la formation des enseignants: une introuvable synchronisation. Université de Genève, Faculté de psychologie et des sciences de l'éducation.

PERRENOUD Ph. (1993): Curriculum : le réel, le formel, le caché, in HOUSSAYE J. (dir.) La pédagogie : une encyclopédie pour aujourd'hui, Paris, ESF, $2^{\mathrm{e}}$ édition 1994, pp. 61-76.

PROST A. (1992): Éducation, société, politiques. Une histoire de l'enseignement en France, de 1945 à nos jours. Paris : Seuil.

ROHR A. (1998): Philipp Albert Stapfer, eine Biographie. Berne : Lang.

VEYNE P. (1971): Comment on écrit l'histoire. Essai d'épistémologie. Paris : Seuil. 